\title{
Procesos de obtención de ferritas hexagonales tipo $\mathbf{M}^{(\bullet)}$
}

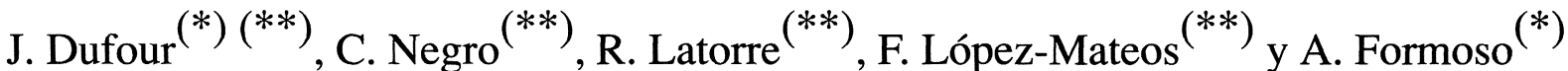

Resumen Actualmente, las ferritas hexagonales tipo $\mathrm{M}$ de bario o estroncio son los imanes permanentes de mayor uso industrial. En este trabajo se revisan los principales métodos de síntesis, haciendo especial hincapié en el método cerámico, ya que es el de mayor importancia industrial: Además, se discuten otra serie de procesos de obtención tales como el vitrocerámico, el de coprecipitación, el de síntesis hidrotérmica y el de síntesis mediante precursores organometálicos. Por último, se discuten las principales aplicaciones industriales de estos materiales.

Palabras clave: Hexaferritas tipo M. Imanes permanentes. Método cerámico. Métodos de síntesis. Aplicaciones.

\section{Obtaining processes of M-type hexaferrites}

\begin{abstract}
M-type barium- or strontium-hexaferrites strontium are the permanent magnets with main industrial use. In this paper, the main synthesis methods are reviewed, special attention being paid to the ceramic method which is the main industrial process. Other obtaining proceedings are also discussed (vitreous-ceramic, coprecipitation, hydrothermal synthesis of organometallic precurssors). Finally, the most important industrial applications are discussed.
\end{abstract}

Keywords: M-type hexaferrites. Permanent magnets. Ceramic method. Synthesis methods. Applications.

\section{INTRODUCCIÓN}

En la actualidad, las hexaferritas tipo $\mathrm{M}$ copan casi totalmente el mercado de imanes permanentes. Su uso aumentó desde un $27 \%$ en 1960 a un $97 \%$ en 1990.

Las hexaferritas tipo $\mathrm{M}$, de fórmula $\mathrm{MFe}_{12} \mathrm{O}_{19}$ $\left(\mathrm{MO} \cdot 6 \mathrm{Fe}_{2} \mathrm{O}_{3}\right.$ ), donde $\mathrm{M}=\mathrm{Ba}$, Sr ó $\mathrm{Pb}(\mathrm{II})$, poseen la estructura cristalina de la magnetoplumbita caracterizada por el grupo espacial $\mathrm{P}_{3} / \mathrm{mmc}$. Está formada por un par de unidades $\mathrm{M}$ rotadas $180^{\circ}$ una respecto a la otra sobre el eje $c$, teniendo, por lo tanto, diez capas. Se trata de un empaquetamiento cerrado de iones oxígeno y bario con cationes férri-

(•) Trabajo recibido el día 13 de junio de 1994

(*) Dpto. de Metalurgia Primaria y Reciclado de Materiales. Centro Nacional de Investigaciones Metalúrgicas. CSIC. Avda. Gregorio del Amo, 8. 28040-Madrid (España).

(*) Dpto. de Ingeniería Química. Facultad de Ciencias Químicas. Universidad Complutense de Madrid. Avda. Complutense, s/n. 28040-Madrid (España). cos en las cinco posiciones intersticiales posibles: $4 f_{1}$ y $4 f_{2}$ (tetraédricas), $2 a$ y $12 k$ (octaédricas) y $2 b$ (bipiramidal) (1).

$\mathrm{El}$ magnetismo de las ferritas $\mathrm{MFe}_{12} \mathrm{O}_{19}$ se debe a los iones $\mathrm{Fe}(\mathrm{III})$, portadores de un momento de $5 \mu_{\mathrm{B}}$; estos se alinean paralela o antiparalelamente por interacción de canje. Cuando los espines se encuentran en paralelo, todos los cationes de la misma posición cristalográfica constituyen una submatriz ferromagnética. La interacción entre iones vecinos de submatrices diferentes se debe a supercanje mediante el oxígeno. La teoría predice que los momentos iónicos se inclinan a ser antiparalelos cuanto más se aproxima a $180^{\circ}$ el ángulo $\mathrm{Fe}-\mathrm{O}-\mathrm{Fe}$ y menor sea la distancia Fe-O-Fe (2). La configuración resultante más probable conduce a un momento magnético neto por celdilla de ocho momentos iónicos de $\mathrm{Fe}(\mathrm{III})$, que son $40 \mu_{B}$, valor que se corresponde con medidas experimentales.

La imanación está fuertemente limitada al eje $c$ hexagonal debido a la alta anisotropía magnetocristalina originada por el acoplamiento espín-órbita de 
los iones hierro (3) y caracterizada por la constante de anisotropía $K_{1}\left(K_{1}=3,3 \cdot 10^{5} \mathrm{~J} \cdot \mathrm{m}^{-3}\right.$ para $\mathrm{BaFe}_{12} \mathrm{O}_{19}$ y $K_{1}=3,6 \cdot 10^{5} \mathrm{~J} \cdot \mathrm{m}^{-3}$ para $\left.\mathrm{SrFe}_{12} \mathrm{O}_{19}(4)\right)$. Las constantes de orden mayor $\left(K_{2}, K_{3}\right)$ son despreciables (5). Los iones $\mathrm{Fe}(\mathrm{III})$, en cada una de las posiciones de la estructura, presentan una contribución diferente a la anisotropía magnetocristalina (6). Estos valores de la constante de anisotropía se ven afectados tanto por la temperatura (7) como por las sustituciones (8).

El comportamiento magnético de las ferritas $\mathrm{M}$ $\mathrm{y}$, en general, de todos los materiales fuertemente magnéticos, se caracteriza por los valores de remanencia $\left(B_{\mathrm{r}}=0,20-0,45 \mathrm{~T}\right)$, coercitividad intrínseca $\left(H_{\mathrm{cj}}=190-360 \mathrm{kA} / \mathrm{m}\right)$ y el producto de energía magnetoestática $\left((B H)_{\operatorname{máx}}=4-34 \mathrm{~kJ} / \mathrm{m}^{3}\right)(9$ y 10$)$, siendo el campo coercitivo uno de los principales. Su generación puede realizarse mediante dos mecanismos (11): rotación coherente, en la que un espín gira por efecto del campo magnético aplicado y arrastra en su giro al espín vecino, produciéndose un efecto en cadena que da lugar a una especie de onda; y por desplazamiento de paredes, que da lugar, por último, a los cristales saturados. Estos dos efectos pueden producirse individualmente o contribuir ambos al campo coercitivo. Asimismo, el campo coercitivo se ve muy afectado por la presencia de imperfecciones que pueden modificar los dominios magnéticos (12).

Para la síntesis de ferritas hexagonales tipo $\mathrm{M}$, existen en la actualidad diversos métodos que se pueden encuadrar en los siguientes grandes grupos.

\section{PROCESOS CERÁMICOS}

El método más común e importante desde el punto de vista tecnológico es el proceso cerámico estándar. En éste, el primer paso consiste en mezclar las materias primas (normalmente óxidos de hierro con carbonatos u óxidos de bario, estroncio o plomo) y someterlas a molienda con el fin de homogeneizar la mezcla. Esta operación puede llevarse a cabo en húmedo, utilizando normalmente agua como portador, aunque se han realizado estudios con otros fluidos (13), o en seco. Inicialmente, los óxidos férricos utilizados eran de alta pureza, pero, debido al gran consumo de este tipo de óxido en la fabricación de la hexaferrita $(86 \%)$ y al relativamente bajo precio de ésta, se han ido reemplazando por materias primas de menor pureza. En la actualidad se emplean óxidos obtenidos mediante la tostación de lejías clorhídricas de decapado (método Ruthner) (14 y 15), óxidos sintéticos producidos por el proceso Lurgi (16), óxidos procedentes de siderurgia (17) y óxidos minerales.

En el caso del procesado en seco, la homogeneización de las materias primas dosificadas se realiza en mezcladores de alto rendimiento, mediante la aplicación de altas velocidades de giro o en molinos, especialmente para la compactación de mezclas en las que se utilizan óxidos tostados por pulverización. La elección entre mezclado en húmedo o mezclado en seco depende del estado original de las materias primas y de las especificaciones del producto final. Para llegar al rango de tamaño superfino es necesario minimizar la aglomeración y la agregación, siendo para ello más fácil trabajar en medio húmedo (18). Un factor importante a considerar es que diversos materiales no son aptos para el proceso de mezclado con la finura requerida y deben molerse durante la propia mezcla. Por lo tanto, debe distinguirse entre la molienda en húmedo real sobre lechos de molienda por agitación o vibración y el mezclado en húmedo en mezcladores de alta velocidad. Si las materias primas no pueden desmenuzarse por mezclado deben someterse a molienda en húmedo. Este último caso se aplica, especialmente, a óxidos minerales con un tamaño de partícula muy grande, óxidos obtenidos mediante tostación de lejías de decapado clorhídricas en lecho fluidizado o cascarilla de laminación (17).

La segunda etapa de este proceso consiste en una presinterización o calcinación. Esta operación es especialmente crítica, ya que determina en gran medida las propiedades finales del producto acabado. El rango de temperaturas de operación se fija entre 900 y $1.300{ }^{\circ} \mathrm{C}$, valores entre los que las materias primas reaccionan completamente para adquirir la estructura de la magnetoplumbita. Si la calcinación se realiza a temperaturas inferiores, se produce un material demasiado blando que en la posterior molienda proporcionará un tamaño de partícula demasiado fino. Por el contrario, si la temperatura es demasiado alta, las partículas serán muy duras y, tras la molienda, la distribución de tamaños de partícula será muy amplia y conducirá a un entrelazamiento y a una fuerza coercitiva muy bajos en el posterior sinterizado (19).

La síntesis de hexaferrita de bario pasa por la formación de monoferrita:

$$
\mathrm{BaCO}_{3}+\mathrm{Fe}_{2} \mathrm{O}_{3} \rightarrow \mathrm{BaFe}_{2} \mathrm{O}_{4}+\mathrm{CO}_{2}
$$

En este paso afectan tanto el tamaño de partícula de las materias primas como la naturaleza de estos óxidos de partida y la presencia de óxidos extraños.

El segundo paso es la formación de la hexaferrita:

$$
\mathrm{BaFe}_{2} \mathrm{O}_{4}+5 \mathrm{Fe}_{2} \mathrm{O}_{3} \rightarrow \mathrm{BaFe}_{12} \mathrm{O}_{19}
$$

En esta etapa afectan el tamaño de partícula, estequeometría y origen de los óxidos férreos, así como los rangos de temperatura (20).

Tras el presinterizado, se realiza una nueva molienda, con el fin de reducir el tamaño de 
partícula a valores de aproximadamente $1 \mu \mathrm{m}$ para obtener propiedades de monodominio.

La conformación del polvo molido puede llevarse a cabo de diferentes maneras, dependiendo del grado de alineamiento magnético deseado. En la forma isótropa en la que las partículas individuales se disponen aleatoriamente unas respecto a otras, resultando las propiedades magnéticas iguales en todas las direcciones, el polvo se seca por pulverización y se comprime en seco para obtener la forma requerida. El conformado es un paso muy importante en la tecnología de fabricación de ferritas. La resistencia mecánica de las muestras depende, fundamentalmente, de los aditivos orgánicos introducidos en el polvo antes del compactado. Los ligantes (binders) y los plastificadores son los dos aditivos más importantes que, posteriormente, se eliminan por quemado. La función principal del ligante (normalmente, un polímero) es facilitar la compactación del polvo para mejorar la resistencia mecánica de las muestras en verde. Además, debe quemarse fácilmente sin contaminar las piezas finales. Como el compactado se suele realizar a temperatura ambiente, se necesita disminuir la temperatura de transición vítrea, $T_{\mathrm{g}}$, del ligante, es decir se precisa tener el polímero en estado fluido, a cuyo efecto se adiciona un plastificante, que puede ser una molécula pequeña o un polímero de peso molecular menor que el del ligante (21).

Los imanes anisótropos, de mejores propiedades, se preparan por conformado en seco o en húmedo en presencia de un campo magnético externo que produce que las partículas se alineen en una dirección. Con el conformado en húmedo se alcanza un mayor grado de alineamiento, ya que, al no estar el material sometido a la fricción entre partículas del proceso en seco, las partículas están mucho más libres para rotar bajo la influencia del campo. Cuando se ha alcanzado el grado de compactación requerido se extrae el agua mediante vacío evitando el desmenuzamiento de la pieza. Se puede evitar la presencia del campo magnético durante el conformado, aplicándolo en la operación de secado (22).

A continuación puede realizarse el sinterizado de las piezas conformadas. No obstante, se deben secar cuidadosamente las piezas compactadas en húmedo para extraer toda la humedad residual antes de introducirlas en el horno.

Las piezas formadas se sinterizan al aire entre 1.100 y $1.400{ }^{\circ} \mathrm{C}$ para producir un material cerámico denso. A fin de minimizar el crecimiento de grano, la temperatura debe mantenerse lo más baja posible (23). Este tratamiento térmico requiere largos períodos de calefacción. En la actualidad, se están realizando distintos estudios para reducir estos tiempos; entre ellos destaca el estudio sobre nuevos diseños de hornos o el tratamiento de sinterizado por microondas (24).
Las etapas de calcinación, molienda y compactación húmeda en presencia de campo magnético, se pueden evitar mediante la compactación de los constituyentes anisótropos y posterior reacción topotáctica, que consiste en la difusión de los iones $\mathrm{Ba}^{2+}$ ó $\mathrm{Sr}^{2+}$ y $\mathrm{O}^{2-}$ dentro de la estructura del $\alpha-\mathrm{Fe}_{2} \mathrm{O}_{3}(25)$.

La sinterización ejerce una influencia muy importante sobre las propiedades magnéticas finales de la hexaferrita tipo M. Como ya se expuso anteriormente, las especificaciones para un material magnético duro vienen determinadas por los valores del par $\left(B_{\mathrm{r}}, H_{\mathrm{cj}}\right)$. La remanencia está gobernada por la imanación intrínseca $\left(J_{\mathrm{s}}\right)$ del material, pero también es una variable extensiva a través de la relación (26):

$$
B=p A J_{\mathrm{s}}
$$

donde $A$ es el factor de orientación que varía entre 0 y 1 dependiendo del alineamiento del cristal, de la simetría cristalográfica y del eje de medida. El factor $p$ es la fracción de empaquetamiento, relacionada directamente con la porosidad de la muestra. En consecuencia, la etapa de sinterización en el proceso de fabricación debe encaminarse a fracciones de empaquetamiento muy alto, cerca del límite teórico de la unidad, para incrementar la remanencia y el producto de energía.

La coercitividad es, también, muy sensible a los procesos de sinterización, estando íntimamente relacionada con la existencia de los dominios. Si se parte de un cuerpo completamente saturado, sin muros de Bloch, la imanación inversa está relacionada con las formas en las que las paredes de los dominios se pinean y se mueven. Partículas menores de $500 \AA$ son superparamagnéticas y no presentan coercitividad a través de la activación térmica de la imanación inversa. Para tamaños mayores, alrededor de 1.000 $\AA$, existe un rango de partículas monodominio donde no puede inducirse una estructura de dominios, ya que la liberación de energía magnetoestática es incapaz de compensar el aumento de las energías de intercambio y magnetocristalina. Para partículas aún mayores existe una región de monodominio inestable, que se alcanza tras imanación total.

La sinterización ejerce dos efectos sobre la coercitividad: se produce un aumento de la misma al restaurar las estructuras desorganizadas y al limpiar la superficie de grano, pero, por incremento del tamaño de grano, los desajustes o nuevos defectos pueden ser más efectivos sobre mayores volúmenes, decreciendo la coercitividad. En general, el segundo efecto aparece a mayores temperaturas, cerca del límite de densidad superior (27).

También se ha estudiado el efecto de aditivos en esta síntesis. Los compuestos utilizados han sido $\mathrm{SiO}_{2}, \mathrm{Al}_{2} \mathrm{O}_{3}, \mathrm{Bi}_{2} \mathrm{O}_{3}$, cloruros y fluoruros. 
El uso de sílice provoca una inhibición del crecimiento de grano en las ferritas. Este fenómeno puede atribuirse a la presencia de trazas de impurezas por segregación y/o a la presencia de fases sólidas de impurezas en las uniones múltiples de grano (28).

La sustitución por alúmina se emplea para modificar las propiedades magnéticas: la remanencia decrece y la coercitividad de polarización aumenta. El descenso de la remanencia puede predecirse por la disminución de la polarización de saturación. No obstante, el incremento en el campo coercitivo no puede explicarse sólo mediante el aumento de la fuerza del campo de anisotropía, sino que depende también de la microestructura cristalina, ya que la presencia de $\mathrm{Al}_{2} \mathrm{O}_{3}$ promueve la formación de granos en forma de placa (29).

La presencia de $\mathrm{Bi}_{2} \mathrm{O}_{3}$ origina la formación de ferrita a temperaturas considerablemente bajas. Además, aumenta la velocidad de reacción de tal forma que con tiempos de sinterización cortos ya no se presentan trazas de óxidos de hierro sin reaccionar (30).

En la figura 1 se resume este proceso en un diagrama de bloques.

Junto con los imanes sinterizados, los imanes de ferrita aglomerados con resinas (plastoferritas) han alcanzado importancia industrial y económica. Estos imanes se producen mezclando polvos de ferrita dura con, por ejemplo, goma, polivinilcloruros, polialcoholes vinílicos (31), poliamidas u otros polímeros (32 y 33). Para ello se pueden utilizar mezcladores, mezcladores-extrusionadores u homogeneizadores. Posteriormente, se emplea un proceso de enrollamiento y moldeo por inyección o por compresión. En la figura 2 se recoge un diagrama de bloques que refleja este proceso.

Se pueden conseguir imanes anisótropos si en la operación de moldeo se aplica un campo magnético orientado.

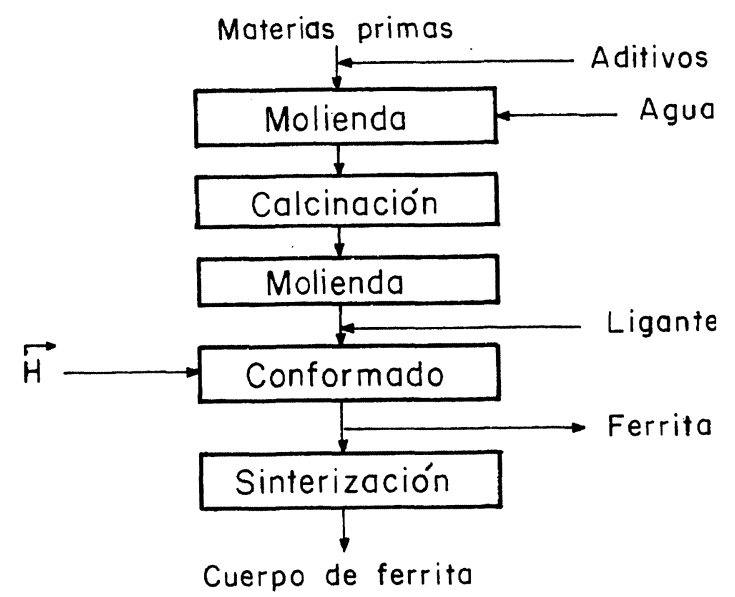

Fig. 1.- Síntesis cerámica de hexaferritas.

FIG. 1.-Ceramic synthesis of hexaferrites.

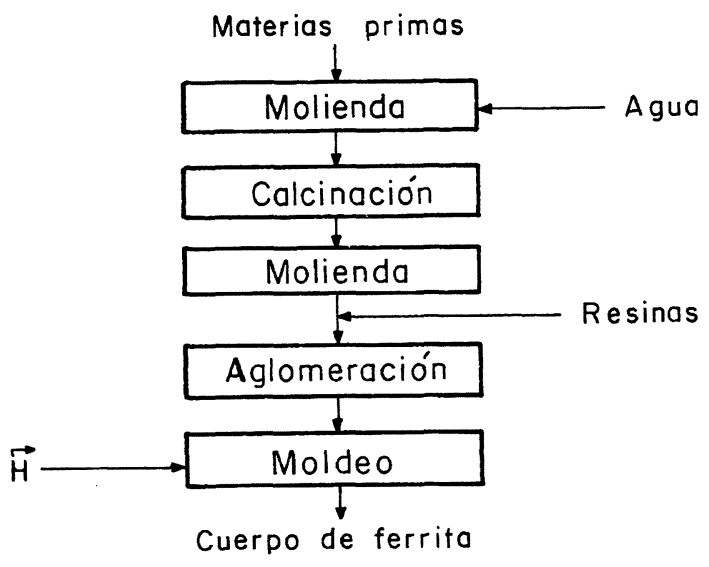

FIG. 2.- Síntesis de plastoferritas.

Fig. 2.-Synthesis of plastoferrites.

Dependiendo del proceso de producción, pueden fabricarse plastoferritas con diferentes contenidos de material magnético. El moldeo por inyección hace posible la obtención de imanes isótropos o anisótropos con un contenido de polvo de ferritas del 50 al $65 \%$ en volumen y mediante moldeo por compresión se consiguen productos con un contenido superior al $80 \%$.

La reducción de las propiedades magnéticas debido a la menor fracción de fase magnética se contrarresta con ciertas ventajas técnicas y económicas. Por ejemplo, las plastoferritas presentan mejores propiedades mecánicas debido a su elasticidad y a su tenacidad (34).

\section{SÍNTESIS VITROCERÁMICA}

Este método se utiliza, principalmente, para la obtención de monocristales. El crecimiento de monocristales de $\mathrm{MFe}_{12} \mathrm{O}_{19}(\mathrm{M}=\mathrm{Ba}, \mathrm{Sr}$ ó $\mathrm{Pb}) \mathrm{a}$ partir de $\mathrm{BaO}$ y $\mathrm{Fe}_{2} \mathrm{O}_{3}$ por fusión es dificultoso, siendo necesario trabajar a más de $1.500{ }^{\circ} \mathrm{C}$ y a presión elevada (35).

El punto de fusión puede disminuirse aumentando la proporción de bario o mediante la adición de otros óxidos, de tal forma que se pueden obtener ferritas libres de $\mathrm{Fe}$ (II) a presión atmosférica. Estos óxidos pueden ser $\mathrm{Na}_{2} \mathrm{O}$ ó $\mathrm{B}_{2} \mathrm{O}_{3}$ (35 y 36). De éstos, las fusiones con óxido de boro presentan más ventajas ya que tanto sus valores de volatilidad y viscosidad como sus puntos de fusión son bajos.

Se puede considerar que la fase vítrea está compuesta por un vitrificante $\left(\mathrm{B}_{2} \mathrm{O}_{3}\right)$, un modificador $(\mathrm{BaO})$ y óxido férrico. La calcinación provoca la cristalización de la hexaferrita de bario en una estructura vítrea formada por el modificador y el vitrificante. Se considera que la fase vítrea es el medio reactivo, similar a una disolución en la que la movilidad de los átomos es reducida (37). 
No se puede trabajar con altos contenidos de hierro, ya que pueden provocar la desvitrificación durante el enfriamiento, aunque con bajos contenidos de dicho elemento, durante la calcinación, se forma $\mathrm{Fe}_{2} \mathrm{O}_{3}$ además de la hexaferrita (38).

El procedimiento de obtención consiste en la fusión de los componentes básicos de la ferrita y de un compuesto de boro (borato sódico, ácido bórico u óxido) como formador vítreo, siendo la temperatura requerida del orden de $1.350{ }^{\circ} \mathrm{C}$. Posteriormente, la mezcla se somete a un enfriamiento rápido con el fin de obtener cristales completamente vitrificados con alto contenido de $\mathrm{Fe}_{2} \mathrm{O}_{3}$. La siguiente etapa consiste en una calefacción entre 500 y 900 ${ }^{\circ} \mathrm{C}$ para inducir la formación de la ferrita. Por último, se trata con ácido acético para eliminar la matriz vítrea $(37,39$ y 40$)$.

Esta vía vitrocerámica de producción de hexaferrita presenta la ventaja de suministrar una mezcla íntima de iones a nivel atómico, con la subsecuente nucleación y crecimiento a menores temperaturas. Simultáneamente, este método proporciona un crecimiento a gran escala del grano que es mucho más común en la síntesis cerámica (41). Como inconveniente, presenta la necesidad de trabajar a temperaturas muy superiores a los métodos cerámicos convencionales para conseguir la fusión.

Se puede utilizar siembra para favorecer la nucleación de la ferrita, empleando una hexaferrita amorfa de estructura indefinida (42).

También, aprovechando el alto poder de disolución de los cloruros alcalinos y alcalinotérreos en estado fundido para el óxido férrico, se pueden crecer grandes monocristales (43). En la figura 3 se recoge el diagrama de flujo de este procedimiento de crecimiento de monocristales.

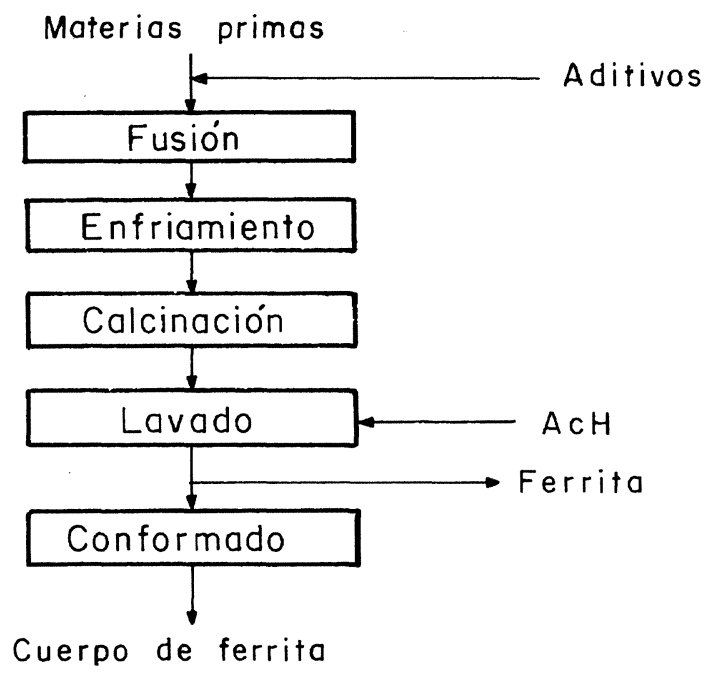

FIG. 3.- Síntesis vitrocerámica de hexaferritas.

FIG. 3.-Vitreous-ceramic synthesis of hexaferrites.

\section{MÉTODOS DE COPRECIPITACIÓN}

Este método consiste en agitar una mezcla de cloruros de $\mathrm{Fe}(\mathrm{III}), \mathrm{Ba}$ ó $\mathrm{Sr}$ (pudiendo incluirse, además, otros metales como titanio, cobalto o samario para mejorar las propiedades (44 y 45)), en una disolución acuosa de hidróxido o de carbonato sódico, o bien sobre otro tipo de base, en concentración mayor a 3 molar y trabajando a una temperatura entre 20 y $90{ }^{\circ} \mathrm{C}$. Así se produce la coprecipitación de los óxidos de hierro y del metal empleado íntimamente mezclados.

La segunda etapa de obtención pasa por la filtración del producto de la coprecipitación y posterior secado a una temperatura inferior a $100{ }^{\circ} \mathrm{C}$.

El polvo obtenido se calcina a $600-1.000{ }^{\circ} \mathrm{C}$ durante 1-6 h. En esta etapa la ferrita puede aparecer impurificada por una pequeña cantidad de $\alpha$ $\mathrm{Fe}_{2} \mathrm{O}_{3}$ que se elimina por lavado con ácido clorhídrico diluido. Pueden alcanzarse partículas con un diámetro cercano a $0,2 \mu \mathrm{m}$ dependiendo del tratamiento térmico y de las condiciones de coprecipitación.

Por último, el sólido obtenido se somete a compactación y sinterización de forma similar a la empleada en el método cerámico.

En la figura 4 se incluye un diagrama de bloques de este proceso.

Cuando se utiliza una mezcla de carbonato e hidróxido sódicos, el producto de la coprecipitación es una mezcla ultrafina de $\mathrm{Fe}(\mathrm{OH})_{3}$ y $\mathrm{MCO}_{3}(\mathrm{M}=$ $\mathrm{Ba}, \mathrm{Sr}$ ). La ferrita se forma a partir de $750^{\circ} \mathrm{C}$, descomponiéndose el carbonato a esta temperatura. Según aumenta el tiempo de calcinación a una temperatura fija, aumenta la pureza de la ferrita.

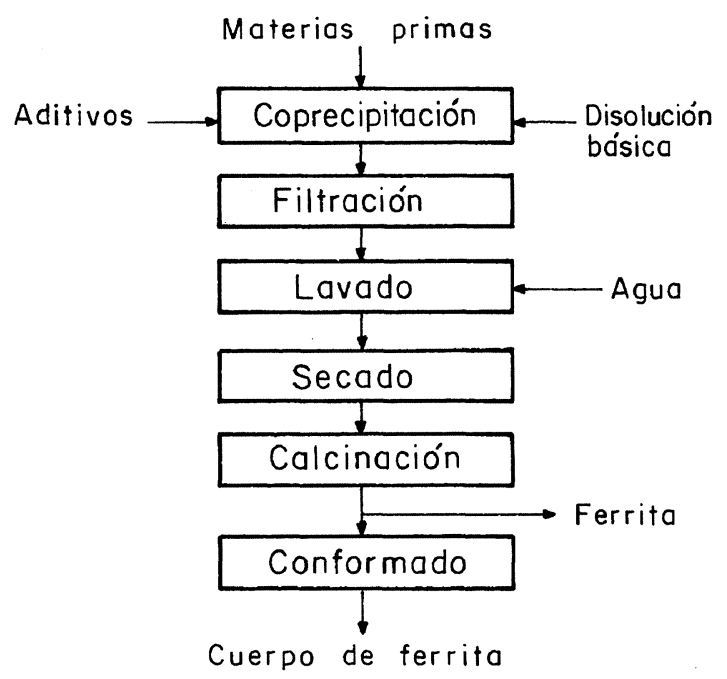

FIG. 4.- Síntesis de hexaferritas por coprecipitación.

FIG. 4.- Synthesis of hexaferrites by coprecipitation 
Otro método de trabajo para modificar las propiedades magnéticas consiste en dejar gotear una disolución de cloruros férrico y de bario o estroncio, que puede contener cloruros de otros metales, sobre una disolución básica de elevado $\mathrm{pH}$ (46).

En el mecanismo de reacción no se forman las fases intermedias $\mathrm{MFe}_{2} \mathrm{O}_{4}, \mathrm{M}_{2} \mathrm{Fe}_{2} \mathrm{O}_{5}$ ó $\mathrm{MFeO}_{3-\mathrm{x}}$ $(\mathrm{M}=\mathrm{Ba}, \mathrm{Sr})$ que se producen en el método cerámico tradicional. Según avanza la reacción, el coprecipitado cambia a la fase $\alpha-\mathrm{Fe}_{2} \mathrm{O}_{3}$ a $650{ }^{\circ} \mathrm{C}$ y se convierte finalmente en $\mathrm{MFe}_{12} \mathrm{O}_{19}(\mathrm{M}=\mathrm{Ba}, \mathrm{Sr}) \mathrm{a}$ unos $800{ }^{\circ} \mathrm{C}$ (47).

Este procedimiento presenta las siguientes ventajas (48):

- Formación de partículas ultrafinas uniformes de gran área superficial.

- Si se comienza con disoluciones bien mezcladas, se alcanza homogeneidad química a escala atómica.

- Se obtienen materiales de alta pureza y libres de tensiones que no necesitan molienda.

- Es posible una conformación conveniente de las partículas trabajando a temperaturas relativamente bajas.

- Estas ferritas son mucho más reactivas y se sintetizan a temperaturas inferiores a las del método cerámico.

Los parámetros de funcionamiento de las ferritas obtenidas por este método parecen ser superiores en un $20 \%$ a los de las ferritas preparadas por el método cerámico convencional (49).

Con un método que combina las ventajas de los procesos de coprecipitación y de fusión de sales, se pueden obtener productos finamente divididos de fuerza coercitiva muy alta. Así, cuando se calientan coprecipitados que contienen cloruro sódico, se obtienen preparaciones monofásicas de ferrita de bario con elevados valores de sus propiedades magnéticas y con pequeños diámetros de partícula. Las partículas cristalizan en una matriz de $\mathrm{NaCl}$ como una monodispersión con un pronunciado hábito hexagonal y una distribución de tamaños de partícula estrecha, encontrándose cada placa separada de sus vecinas por un estrato de sal. Después de disolver el cloruro sódico con agua, se obtiene un polvo muy finamente dividido, sin sinterizar y muy dispersable, con especial interés para pigmentos magnéticos (35 y 50).

\section{MÉTODOS DE SÍNTESIS HIDROTÉRMICA}

En este procedimiento se prepara una dispersión de óxido, hidróxido u oxihidróxido de $\mathrm{Fe}(\mathrm{III})$ en una disolución acuosa de hidróxido bárico (51). La utilización de $\alpha$-FeOOH mejora las preparaciones de ferrita (52).

Posteriormente, se realiza la reacción a presión en autoclave a una temperatura entre 250 y $350{ }^{\circ} \mathrm{C}$ con una velocidad de calefacción lenta $\left(<5^{\circ} \mathrm{C} / \mathrm{min}\right)$. El tiempo de reacción oscila entre 15 y 25 h, utilizando atmósfera oxidante con el fin de prevenir la posible reducción del óxido férrico por el hidrógeno formado en la reacción del agua con el acero inoxidable del reactor. El producto obtenido se lava con disoluciones ácidas de baja concentración hasta eliminación de iones $\mathrm{Fe}(\mathrm{II})$. Los tamaños de los cristales sintetizados se encuentran en el rango de partículas monodominio (53).

Por último se realiza la conformación de la pieza y se sinteriza a $1.000{ }^{\circ} \mathrm{C}$, alcanzándose una buena densificación (54).

En la figura 5 se recoge un diagrama de bloques de este procedimiento de síntesis.

Esta síntesis puede complicarse por la aparición de otras fases; por ejemplo en el menor rango de temperatura $\left(<180^{\circ} \mathrm{C}\right)$ se forma la estructura $\mathrm{BaO} \cdot 4,5 \mathrm{Fe}_{2} \mathrm{O}_{3}$ no ferrimagnética, que se consigue también introduciendo un gas oxidante en una suspensión alcalina de $\mathrm{Fe}(\mathrm{OH})_{2}$, que contenga ion bario, obtenida a partir de diferentes sales (por ejemplo, $\mathrm{FeCl}_{2}-\mathrm{BaCl}_{2}-\mathrm{NaOH}$ ) (55).

En contraste con el método cerámico, se obtiene una ferrita finamente dividida y con una distribución de tamaños de partícula comparativamente estrecha. Este método evita las etapas de molienda y compactación. Como inconvenientes, presenta la necesidad de trabajar a altas presiones y el prolongado tiempo de reacción.

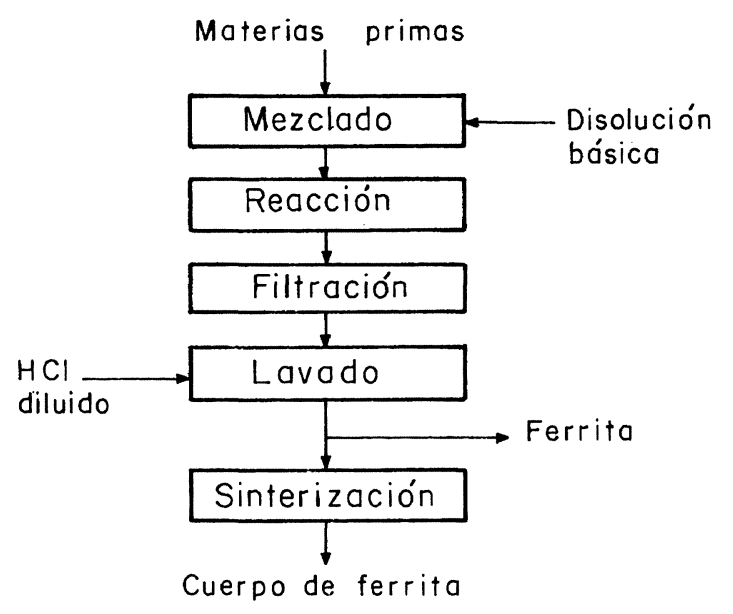

Fig. 5.- Síntesis hidrotérmica de ferritas tipo $\mathrm{M}$.

FIG. 5.- Hydrothermal synthesis of hexaferrites. 
La mayor ventaja de este tipo de síntesis es la producción de una suspensión de óxidos metálicos extremadamente finos que, en algunos casos, puede usarse para el método cerámico sin el paso de calcinación o presinterización. Además, puede obtenerse un producto con una estructura cristalina perfecta con una composición química definida y que puede depurarse fácilmente mediante un simple lavado (56).

\section{SÍNTESIS POR PRECURSORES ORGANOMETÁLICOS}

Este tipo de síntesis consiste en la formación de precursores organometálicos (malonatos, hidracinacarboxilatos hidratados, citratos, compuestos piridínicos, etc.) de hierro y bario o estroncio (57 y 58), que tras secado se calcinan en un rango de temperaturas que oscila entre los 900 y los $1.000{ }^{\circ} \mathrm{C}$ para producir la hexaferrita.

Para obtener los precursores se mezcla una disolución acuosa de sales de los metales seleccionados (nitratos, cloruros o sulfatos, normalmente) con disoluciones del compuesto orgánico con el que se desea sintetizar el organometálico. Además, se adiciona una base para incrementar el $\mathrm{pH}$ y facilitar la formación de una disolución homogénea. Posteriormente se calienta hasta evaporación del agua (59) o hasta eliminación de la base excedente, precipitando el sólido, en este caso, por goteo en etanol (60). Por último, el sólido se filtra y se lava.

En la figura 6 se presenta un diagrama de bloques representativo de este proceso de síntesis de hexaferrita.

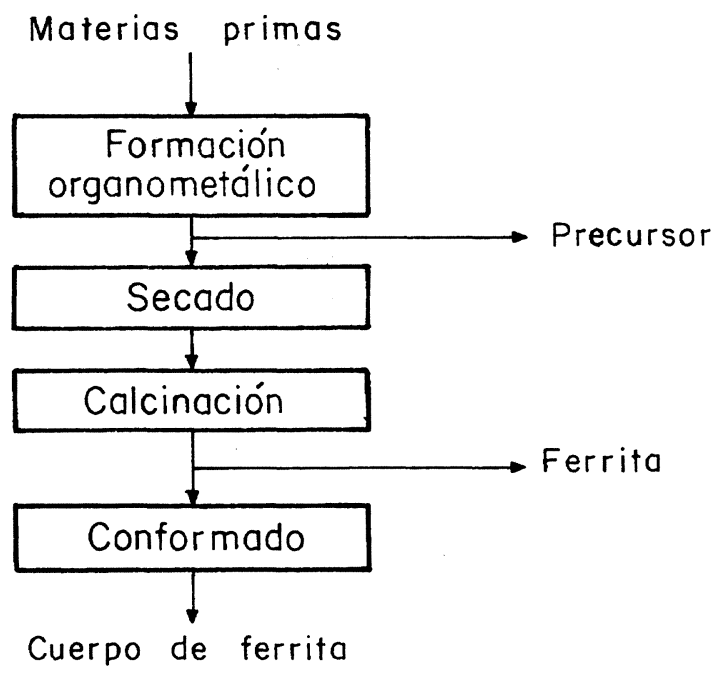

FIG. 6.- Obtención de ferritas mediante síntesis de organometálicos.

FIG. 6.- Obtaining of ferrites meaning organometallics synthesis.
Este método parece estar más indicado para la síntesis de ferritas de tipo espinela (59 y 61). También se ha logrado la síntesis de fases de ferrita intermedias en la obtención de la hexaferrita (62).

Existe, además, una serie de métodos experimentales para la síntesis de hexaferritas tipo M, pero que todavía no han encontrado aplicación industrial. Entre ellos, se pueden citar: método solgel (63), obtención de películas de ferrita mediante pirólisis (64), pirólisis de un aerosol (65), síntesis a baja temperatura mediante cristalización a partir de un gel (66), método del precursor (67), método de combustión (68), etc.

\section{APLICACIONES}

Las principales propiedades de las ferritas hexagonales son su bajo precio, baja densidad y su alta fuerza coercitiva (69). Debido a su bajo coste, las ferritas han reemplazado a otros materiales magnéticos en sistemas ya existentes, como, por ejemplo, en altavoces.

Dado el alto campo coercitivo de este tipo de imanes, las hexaferritas $M$ se utilizan especialmente en motores de corriente continua, especialmente dentro de la industria del automóvil (en elevadores de ventanilla, en motores de limpiacristales o en motores de starter). A estos productos se destina el $70 \%$ de la producción total. Parece ser que la producción a gran escala se concentra en sistemas que requieren imanes relativamente grandes. El resto de la producción se distribuye en un amplio rango de aplicaciones, tales como máquinas de afeitar, mezcladores de café y en otra serie de pequeños electrodomésticos (69).

Otras aplicaciones de las hexaferritas $\mathrm{M}$, aparte del campo de los imanes permanentes, se encuentran en registro en cinta magnética y magnetoóptica. Estas ferritas tipo $M$ son de interés para dispositivos de microondas de tipo resonante (aisladores autorresonantes, filtros y circuladores). Asimismo, se emplean también como componentes magnéticos de polarización en memorias magnéticas de burbuja. Los dominios magnéticos de burbuja son dominios reversos cilíndricos en un cristal fino con su eje dominante preferenciado normal a la superficie.

Actualmente, aprovechando que la dirección de fácil imanación es perpendicular al plano de las placas, se utilizan como medios de registro vertical (70 y 71). También, la dirección vertical de la imanación las hace extremadamente apropiadas para registro de alta densidad o de onda corta (72). Además, la hexaferrita de bario encuentra aplicaciones en registro digital (73), en duplicación de contacto magnético para R-DAT (74), en registro de audio y vídeo, para almacenamiento de datos y para discos 
blandos (floppy disks) y en registro de alta densidad de casetes compactas. Sin embargo, la alta coercitividad de la ferrita pura la convierte en inapropiada como medio de registro magnético y debe reducirse antes de que pueda usarse para el almacenamiento de audio, de vídeo o de datos (75).

Por su parte, las plastoferritas, debido a sus propiedades especiales, han encontrado aplicación en cierres estancos (neveras), juguetes, imanes correctores de tubos catódicos, etc. También se ha estudiado su posible aplicación en almacenamiento de información (76).

En España, la oferta de materiales magnéticos procede de empresas de pequeño tamaño que completan su catálogo con productos importados, existiendo una pequeña producción de imanes permanentes. No obstante, no existe en nuestro país fabricación de ferritas duras basadas en óxidos de bario o de estroncio y la producción de ferritas blandas se encuentra localizada en una sola fábrica perteneciente a una empresa multinacional que destina el $97 \%$ de su producción a la exportación (77). En la figura 7 se presenta el reparto del mercado nacional de materiales magnéticos y sus consumos estimados para 1995.

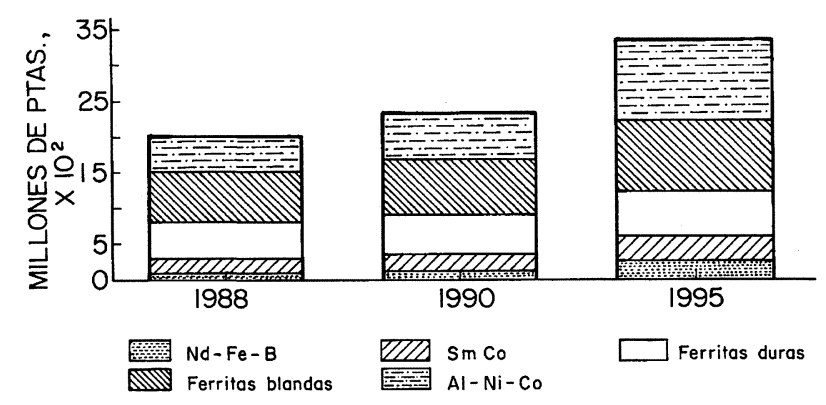

FIG. 7.- Mercado nacional de imanes permanentes.

FIG. 7.- Spanish home market of permanent magnets.

\section{REFERENCIAS}

(1) Smit, J. y WiJn, H.P.J. Ferrites. Ed. John Wiley \& sons. Nueva York, 1959.

(2) Kools, F. Encyclopedia of Science Materials and Engineering. Ed. Pergamon Press. Nueva York, 1986: 2082-2088

(3) Sánchez Girón, V. Materiales magnéticos. Ed. Montecorvo, Madrid, 1965.

(4) McCurrie, R.A. y JaCKson, S. J. Appl. Phys. 61 (10), 1987: 4858-4861.

(5) Graham, C.D. Encyclopedia of Science Materials and Engineering. Ed. Pergamon Press. Nueva York, 1986: 2689-2692

(6) You, X., Daping, Ch., Guiling, Y. y Hongru, Z. Proc. ICF-5. India, 1989: 485-489.

(7) Diao, Z. Proc. ICF-5. India, 1989: 479-483.
(8) Pollert, E. Prog. Crystal Growth Charact. Vol. 11 1985: 155-205.

(9) JILES, D. Introduction to magnetism and magnetic materials. Ed. Chapman and Hall. Suffolk (Gran Bretaña), 1991.

(10) MCCURRIE, R.A. Ferromagnetic Materials. Structure and properties. Ed. Academic Press Inc. Londres, 1994: 243244

(11) ElK, K. y JAHN, L. Phys. Stat. Sol. (b) 168, 1991: 591596.

(12) WANG, F.F.Y. IEEE Trans. Mag. Vol. MAG-21 No. 5, 1985: 1889-1890.

(13) Dufour, J., Latorre, R., Negro, C., Alcalá, E.M., López-Mateos, F. y Formoso, A. Proc. International Conference on Magnetism, Varsovia, 1994: 451

(14) Wurmbauer, D. Wire Ind. enero 1978: 43-45.

(15) RuthNER, M.J. Proc ICF-5. India, 1989: 23-34.

(16) Jewell, C.J. y Marcotte, B.A. Iron Control Hydrometall, 1986: 502-519.

(17) RIES, H.B. Proc. ICF-5. India, 1989: 155-159.

(18) HeInRICh, B., KREITNER, L. y Messer, P.F. Encyclopedia of Science materials and Engineering. Ed. Pergamon Press. Nueva York, 1986: 3055-3059.

(19) GutiÉRreZ, M. y SECO, F.J. Proc. IV Reunión Nacional de Materiales. Oviedo, 1993: 344-345.

(20) HÄDRICH, W. y SLADEK, R. cfi/Ber.DKG 6/7-87, 1987: 230-234.

(21) Morell, A., Eranian, A., Peron, B. y Bezeulin, P. Proc. ICF-5. India, 1989: 137-142.

(22) Hitachi Metals Co. Pat. Japon Kokai Tokkyo Koho Jpn. Pat. 80,157,216. Año 1980.

(23) Lemaire, H. Powder Metall. 25 (3), 1982: 165-172.

(24) Krage, M.K. Ceram. Bull. 60 (11), 1981: 1232-1234.

(25) Gumaste, J.L., NayaK, B.B., Galgali, R.K., Mohanty, B.C. J. Mater. Sci. 23 (1988): 3125-3128.

(26) Martin, D.L. y Benz, M.G. "AIP Conf. Proc. No. 5 Magnetism and Magnetic Materials”. Nueva York, 1972: 970-990.

(27) Dufour, J., Latorre, R., Negro, C., LÓPez, E., AlCalá, E.M., BARBA, C., Formoso, A. y LóPEZ-Mateos, F. Proc. ICEM-13. París 1994: 1191-1192.

(28) Fang, T., Hwang, J.B. y ShiaU, F.S. J. Mater. Sci. Let. 8, 1988: 1386-1388.

(29) Kools, F. y Henket, B. Proc. ICF-5. India, 1989: 417423.

(30) Ram, S., KRIHNAN, H., RaI, K.N. y NARAYAN, K.A. Jpn. J. Appl. Phys. 28 (4), 1989: 604-608.

(31) HimeJ, K.K. Deshi Pat. Japon Kokay Tokkyo Koho Jpn. Pat. 59,174,573. Año 1984.

(32) Fuji Electrochemical Ltd. Co. Pat. Japon Kokai Tokkyo Koho Jpn. Pat. 81,112,702. Año 1981.

(33) Hitachi Metals Co. Pat. Japon Kokai Tokkyo Koho Jpn. Pat. 80, 33,173. Año 1980.

(34) ERVENS, W. y WILMESMEIER, H. Ullmann`s Encyclopedia of Industrial Chemistry. Vol A16. Ed. VCH. Weinheim, 1990: 1-51.

(35) Hibst, H. Angew. Chem. Int. Ed. Engl. 21, 1982: 270282.

(36) HABEREy, F. y KocKel, A. IEEE Trans. Mag. MAG12, 1976: 983-985.

(37) Chaumont, C. J. Phys. IV, 1992: C2/249-C2/252.

(38) Shirk, B.T. y Buessem, W.R. J. Am. Ceram. Soc. 53 (4), 1970: 192-196. 
(39) Kamiyama, K. Patente alemana. Ger. Offen. DE 3,405,603. Año 1984.

(40) KATORI, K. Pat. Japon Kokai Tokkyo Koho Jpn. Pat. 61, 81,608. Año 1986.

(41) Bahadur, D y Chakravorty, D. Proc. ICF-5. India, 1989: 189-193.

(42) Toda Kogyo Corp. Pat. Japon Kokai Tokkyo Koho Jpn. Pat. 58,041,646. Año 1983.

(43) Robinson, T.M. y Labeyrie, M. IEEE Trans. Mag. MAG-23 (5), 1987: 3727-3729.

(44) Endo, H. Patente europea. Pat. Appl. EP 39,773. Año 1981.

(45) Yамамото, A. Pat. Japon Kokai Tokkyo Koho Jpn. Pat. 63,274,628. Año 1988.

(46) Chang, C.W., Tzeng, M.S. y Wang, S.J. J. Mat Sci. Let. 9, 1990: 832-835.

(47) Haneda, K., Miyakawa, C. y Kojima, H. J. Am. Ceram. Soc. 57 (8), 1974: 354-357.

(48) Date, S.K., Deshpande, C.E., Kulkarni, S.D. y SHROTRI, J. J. Proc. ICF-5. India, 1989: 55-59.

(49) Kulkarni, S., Shrotri, J., DeshPande, C.E., Date, S.K. J. Mater. Sci. 24, 1989: 3739-3744.

(50) Odan, K. Pat. Japon Kokai Tokkyo Koho Jpn. Pat. 63,265,829. Año 1988.

(51) Hayakawa, M. Patente alemana. Ger. Offen. DE 3,238,309. Año 1983.

(52) Martín, A. y Carmona, F. Patente española 472,030. Año 1978.

(53) Barb, D., Diamanescu, L., Rusi, A., Tarabasanu, D., Morariu, M. y TeOdorescu, V. J. Mater. Sci. 21, 1986: 1118-1122.

(54) Kormaneni, S., Fregueau, E., Breval, E., Roy, R. $J$. Am. Ceram. Soc. 72, 1988: C26-C28.

(55) NagaI, N. Pat. Japon Kokai Tokkyo Koho Jpn. Pat. 62,052,133. Año 1987.

(56) Wang, M.L., ShiH, Z.W. y Lin, C.H. Ind. Eng. Chem. Res. 31, 1992: 828-833.

(57) Ravindranathan, P. y Patil, K.C. Am. Ceram. Soc. Bull. 66 (4), 1987: 688-692.

(58) Aoki, S. y OzAKI, Y. Pat. Japon Kokai Tokkyo Koho Jpn. Pat. 63,274,628. Año 1987.

(59) RichARDSON, R.T. J. Mater. Sci. 15, 1980: 2569-2572.
(60) Sankaranarayanan, V.K., Pankhurst, Q.A., Dickson, D.P.E. y Johnson, C.E. J. Mag. Magn. Mat. 120, 1993: 73-75.

(61) Mendelovici, E., Villalba, R. y Sagarzazu, A. J. Mater. Sci.. Let. 9, 1990: 28-31.

(62) Bassi, P.S., Randhawa, B.S. y KaUR, S. Proc. ICF-5. India, 1989: 67-71.

(63) Brahma, P., Chakarvorty, D., Singh, K. y Bahadur, D. J. Mater. Sci. Let. 9, 1990: 1438-1440.

(64) Matsumoto, M., Morisako, A. y Hae-Iwa, T. Proc. ICF5. India, 1989: 533-537.

(65) Cabañas, M.V., González-Calbet, J.M., Labeau, M., Mollard, P., Pernet, M. y Vallet-Regi, M. J. Sol. Sta. Chem. 101, 1992: 265-274.

(66) Fan, X.J. y MatiJevic, E. J. Am. Ceram. Soc. 71 (1), 1988: C60-C62.

(67) Ardiaca, R., Ramos, R., Isalgu, A., Rodríguez, J., Obradors, X., Pernet, M. y Vallet, M. IEEE Trans. Mag. MAG-23 (1), 1987: 22-24.

(68) Castro, S., Gayoso, M., RodríGuez, C., Mira, J., Riva, J. y Greneche, J.M. Proc. IV Reunión Nacional de Materiales. Oviedo, 1993: 346-347.

(69) ReYnolds, T.G. Encyclopedia of Chemical Technology Kirk-Othmer. Ed. John Wiley \& Sons. Nueva York, 1980: 881-902.

(70) LiCcI, F., Turilli, G. y Besagni, T. IEEE Trans. Mag. MAG-24 (1), 1988: 593-597.

(71) Matsumoto, K., Yata, M., Nakagaway, S. y NaOe, M. Proc. ICF-5. India, 1989: 507-511.

(72) Appleton, S.G., Pointon, A.J., CobB, A.P.D. y Nixon, D.E. Proc. ICF-5. India, 1989: 507-511.

(73) Speliotis, D.E. J. Appl. Phys. 61, 1987: 3878-3880.

(74) Negaki, T., Chubachi, R., Odaka, K., Shinpuku, Y., TIJIMA, T. y KoriKawA, K. IEEE Trans. Mag. 22, 1986: 1191-1193.

(75) MAThUR, M.C.A., DatTA, S., SPRATT, G.W.D. y KRYDER, M. Proc. ICF-5. India, 1989: 869-872.

(76) FAYLING, R.E. IEEE Trans Mag. MAG-15, 1979: 15671569.

(77) Dirección General de Electrónica y Nuevas Tecnologías (Ministerio de Industria y energía). Plan de Desarrollo Tecnológico en Biotecnologías, Tecnologías Químicas y Materiales Avanzados, 1991: 42-72. 